\title{
Thiamine in diabetic nephropathy: a novel treatment modality? Reply to Alkhalaf A, Kleefstra N, Groenier KH et al. [letter]
}

\author{
N. Rabbani • S. S. Alam • S. Riaz • J. R. Larkin • \\ M. W. Akhtar • T. Shafi • P. J. Thornalley
}

Received: 19 February 2009 / Accepted: 23 February 2009/Published online: 25 March 2009

(C) Springer-Verlag 2009

Keywords Diabetic complications · Diabetic nephropathy · Microalbuminuria $\cdot$ Thiamine . Type 2 diabetes

\section{Abbreviations \\ ACEI Angiotensin-converting enzyme inhibitor \\ ARB Angiotensin receptor blocker \\ RAAS Renin-angiotensin-aldosterone system \\ sVCAM-1 Soluble vascular cell adhesion molecule-1 \\ TPP Thiamine pyrophosphate \\ UAE Urinary albumin excretion}

To the Editor: We thank Alkhalaf and co-authors for their comment on our recent paper in Diabetologia [1]. We are

N. Rabbani $\cdot$ J. R. Larkin $\cdot$ P. J. Thornalley $(\bowtie)$

Clinical Sciences Research Institute, Warwick Medical School,

University of Warwick, University Hospital,

Clifford Bridge Road,

Coventry CV2 2DX, UK

e-mail: P.J.Thornalley@warwick.ac.uk

\section{S. S. Alam}

Department of Pharmacology, Sheikh Zayed Federal Postgraduate Medical Institute, Sheikh Zayed Hospital,

Lahore, Pakistan

\section{S. Riaz}

Department of Microbiology and Molecular Genetics,

University of the Punjab,

Lahore, Pakistan

M. W. Akhtar

School of Biological Sciences, University of the Punjab,

Lahore, Pakistan

T. Shafi

Department of Nephrology, Division of Medicine, Shaikh Zayed Postgraduate Medical Institute,

Lahore, Pakistan grateful for their interest in our work and the recognition of its potential importance in stimulating further research, which may lead to a significant improvement in the treatment of diabetic nephropathy. They have made several comments on issues that could not be dealt with in our short communication [2]. We now address these comments.

The urinary albumin excretion (UAE) data in Table 1 of our paper are the median (minimum-maximum) values of the $24 \mathrm{~h} \mathrm{UAE}$ values for the treatment groups at the study times indicated. The data illustrated in Fig. 1a are the median values of data distributions of $24 \mathrm{~h} \mathrm{UAE}$ change from baseline deduced for each patient. These data sets are not the same: one is absolute UAE values (distributions summarised in Table 1) and the other is of changes in UAE from baseline for each patient (medians of changes shown in Fig. 1a). This, and the non-parametric distribution of the data sets, accounts for the different values for the difference between median UAE after 3 months of treatment and at baseline $(-13.6 \mathrm{mg} / 24 \mathrm{~h})$ that can be deduced from Table 1 and the median of $24 \mathrm{~h}$ UAE changes from baseline $(-17.7 \mathrm{mg} / 24 \mathrm{~h})$ shown in Fig. 1a for the thiamine treatment group. Computing a data distribution of analyte change from baseline is a strategy often employed where there is large dispersion of baseline data, and the response to therapy (rather than change in absolute value) is of greatest interest. Change in UAE was the primary endpoint of our study.

There was a trend for UAE data during the posttreatment washout period to show a continuing decrease beyond that achieved in the treatment period. We stated that the decrease in UAE in the post-treatment period was 'maintained', as the trend for further decrease was not statistically significant. This is not a concern, but rather it might be expected that patients have continued benefit during the washout period from the thiamine supplementa- 
tion in the preceding 3 months. The half-life of thiamine in plasma is relatively short (2 days) [3], whereas the biological half-life of thiamine is much greater (9-18 days) [4]. The plasma concentration and urinary excretion of thiamine of patients in the thiamine treatment arm of the study returned to baseline levels after the 2 month washout period. It is likely, therefore, that increased tissue levels of thiamine pyrophosphate (TPP), and activities of TPPdependent enzymes and related pharmacological responses, remain above baseline levels for at least one biological halflife of thiamine into the washout period of patients in the thiamine treatment group. This may explain the prolongation of beneficial effects of thiamine supplementation into the washout period. Extension of a therapeutic benefit of thiamine into the washout period may have also contributed to the decrease in median plasma soluble vascular cell adhesion molecule-1 (sVCAM1) concentration, which achieved statistical significance only after the washout period. There are additional effects of thiamine therapy that would be expected to extend the period of therapeutic benefit further-such as decreased extracellular matrix expression and deposition by reversal of hyperglycaemia-induced activation of protein kinase $\mathrm{C}$ beta [5]. These effects may give rise to a 'therapeutic memory' of thiamine supplements in diabetes. The maintained therapeutic benefit of thiamine supplements in diabetic nephropathy during a short period of discontinued therapy is an advantage when treatment is inadvertently or unavoidably stopped. Maintained therapeutic benefit during the washout period was a characteristic of high-dose therapy with the angiotensin receptor blocker (ARB) irbesartan ( $\geq 300 \mathrm{mg} /$ day) but not of low-dose therapy (150 mg/day), where a decrease in UAE during treatment was reversed after only 1 month washout [6].

The median UAE of patient groups at baseline in the placebo and thiamine treatment groups were different, but the difference was not statistically significant. This difference was eliminated from the treatment response by deducing the change in UAE from baseline-as presented in Fig. 1a. The effect of baseline UAE on therapeutic outcome was explored further by assessing the link of the treatment response to baseline UAE in correlation analysis. The change in UAE from baseline during the treatment period correlated negatively with baseline UAE for the thiamine treatment $(r=-0.565$, $p<0.001)$ and placebo ( $r=-0.531, p<0.05$; Spearman $)$ groups. The change in UAE did not correlate with any other analyte measured. This correlation has been reported for angiotensinconverting enzyme inhibitor (ACEI) therapy for type 2 diabetic patients with microalbuminuria [7]. For this range of UAE, patients with higher UAE values at baseline showed the greatest response to improved therapy-as seen in the individual responses to thiamine treatment shown in Fig. 1c of our paper. In the thiamine treatment group, all patients had decreased UAE after treatment, supporting previous findings in type 1 diabetes that increased UAE is potentially reversible at this stage of diabetic nephropathy [8].

Alkhalaf and co-authors comment that 'the effects of these drugs on UAE may take a long time to become detectable' and in relation to this they mention use of ACEIs and ARBs. Similar intervention studies with the ARB irbesartan achieved a marked decrease in UAE after 3 months of treatment [6], as employed in our study. The time course effect on UAE is, therefore, typical of effective therapy; both the effectiveness of thiamine supplementation and the time course of the response to supplementation were as expected for translation of our previous pre-clinical studies [5].

The treatment of patients with ACEIs and ARBs in this study was distributed equally between placebo and thiamine treatment groups: six patients in each treatment group. The median baseline UAE of patients treated with thiamine was similar for those with and without ACEI/ARB therapy. After 3 months treatment with thiamine, UAE had decreased significantly in patients with and without ACEI/ ARB therapy. Median UAE values were: patients with thiamine and ACEI/ARB therapy $29.8 \mathrm{mg} / 24 \mathrm{~h}(-33 \%)$ vs $44.8 \mathrm{mg} / 24 \mathrm{~h}$ at baseline $(p<0.05, n=6)$; and patients with thiamine but without ACEI/ARB therapy $30.1 \mathrm{mg} / 24 \mathrm{~h}$ vs $43.7 \mathrm{mg} / 24 \mathrm{~h}(-31 \%)$ at baseline $(p<0.01, n=13$; Wilcoxon signed ranks test). The reader should recall that one patient in the thiamine treatment group was omitted from analysis for suspected illicit thiamine supplementation at baseline. This suggests that the benefits of thiamine supplementation on the renal function of patients with type 2 diabetes with microalbuminuria are available to both those receiving and not receiving $\mathrm{ACEI} / \mathrm{ARB}$ therapy, supporting the importance of pursuing further thiamine supplementation therapy for diabetic nephropathy. Addition of thiamine supplementation therapy may further improve best current therapy.

Alkhalaf and co-authors comment that linear regression of UAE on treatment time was mentioned, and it was not clear what the time unit was and whether the regression slopes differed significantly between the groups. The linear regression was of median change in UAE from baseline (mg/24 h) on treatment period (months) — as was evident in the graph (Fig. 1a) to which the regression statement referred. The regression slopes have units of $\mathrm{mg}^{2} 4 \mathrm{~h}^{-1}$ month $^{-1}$. The regression slopes have no direct link to individual patient responses but were given to quantify the time course plots in Fig. 1a. Hence, the statistical significance indicated in the figure is appropriate and sufficient, in our view.

Alkhalaf and co-authors comment that for the microalbuminuric patients in our study fewer than half were receiving antihypertensive treatment. It is correct that current treatment guidelines advise the use of renin-angiotensinaldosterone system (RAAS) blockade in all type 2 diabetic patients with microalbuminuria [9]. However, a value of $30 \%$ of patients treated with ACEI/ARB therapy is typical 
for the Indian subcontinent - as found similarly in the Chennai Urban Rural Epidemiology Study [10]. Imperfect access to effective treatment in developing countries for socioeconomic reasons, among other reasons, is a contributing factor to the global burden of diabetic nephropathy [11]. This is an area where relatively inexpensive and widely available thiamine supplements may help.

Finally, we agree that this pilot study requires support from further studies, as stated in our trial report. Our study is the first of its kind, however, and shows that thiamine supplements improve renal function in type 2 diabetic patients with microalbuminuria-including for patients receiving RAAS blockade therapy. We thank Dr Alkhalaf and co-authors, and the Editor, for the opportunity to highlight these aspects.

Duality of interest The authors declare that there is no duality of interest associated with this manuscript.

\section{References}

1. Alkhalaf A, Kleefstra N, Groenier KH, Bakker SJL, Navis G, Bilo HGJ (2009) Thiamine in diabetic nephropathy: a novel treatment modality? Diabetologia. doi:10.1007/s00125-009-1326-7
2. Rabbani N, Alam SS, Riaz S et al (2008) High dose thiamine therapy for patients with type 2 diabetes and microalbuminuria: a randomised, double-blind, placebo-controlled pilot study. Diabetologia 52:208-212

3. Weber W, Kewitz H (1985) Determination of thiamine in human plasma and its pharmacokinetics. Eur J Clin Pharmacol 28:213219

4. Ariaey-Nejad MR, Balaghi M, Baker EM, Sauberlich HE (1970) Thiamin metabolism in man. Am J Clin Nutr 23:764-778

5. Babaei-Jadidi R, Karachalias N, Ahmed N, Battah S, Thornalley PJ (2003) Prevention of incipient diabetic nephropathy by highdose thiamine and benfotiamine. Diabetes 52:2110-2120

6. Andersen S, Brochner-Mortensen J, Parving HH (2003) Kidney function during and after withdrawal of long-term irbesartan treatment in patients with type 2 diabetes and microalbuminuria. Diabetes Care 26:3296-3302

7. Chan JCN, Ko GTC, Leung DHY et al (2000) Long-term effects of angiotensin-converting enzyme inhibition and metabolic control in hypertensive type 2 diabetic patients. Kidney Int 57:590 600

8. Perkins BA, Ficociello LH, Silva KH, Finkelstein DM, Warram JH, Krolewski AS (2003) Regression of microalbuminuria in type 1 diabetes. New Engl J Med 348:2285-2293

9. American Diabetes Association (2008) Standards of medical care in diabetes-2008. Diabetes Care 31:S12-S54

10. Unnikrishnan R, Rema M, Pradeepa R et al (2007) Prevalence and risk factors of diabetic nephropathy in an urban south Indian population: the Chennai Urban Rural Epidemiology Study (CURES 45). Diabetes Care 30:2019-2024

11. Luk A, Chan JCN (2008) Diabetic nephropathy-what are the unmet needs? Diabetes Res Clin Pract 82:S15-S20 